\title{
FISIOLOGIA E VIGOR DE SEMENTES DE Mimosa caesalpiniifolia BENTH EM CONDIÇÕES DE ESTRESSE HÍDRICO
}

\author{
Ana Carolina Bezerra ${ }^{1}$, Luana da Silva Barbosa², José Flávio Cardoso Zuza ${ }^{3}$, Camila Firmino de \\ Azevedo $^{4}$
}

\begin{abstract}
RESUMO - Mimosa caesalpiniifolia Benth. é uma espécie promissora para implantação de florestas no Nordeste brasileiro. Contudo, a água é o um fator que exerce influência sobre o processo germinativo afetando diversos processos fisiológicos, como fotossíntese e respiração, bem como a sua produção. Diante do exposto, objetivouse avaliar a germinação e o vigor de sementes de Mimosa caesalpiniifolia Benth. submetidas a diferentes condições de estresse hídrico. As sementes de M. caesalpiniifolia foram coletadas na cidade de São Sebastião de Lagoa de Roça/PB onde foram beneficiadas e homogeneizadas para a retirada de uma amostragem representativa e posteriormente foram submetidas à superação de dormência por meio de desponte na região oposta à micrópila. Logo após, as mesmas foram desinfestadas com hipoclorito de sódio a 0,6\%, durante 5 minutos e lavadas em água corrente por 10 minutos. Para a avaliação das diferentes condições de estresse hídrico, as sementes foram semeadas em bandejas plásticas individuais para cada repetição, contendo areia umedecida com cinco diferentes níveis de capacidade de retenção (10, 20, 30, 40, 50 e 60). As variáveis, germinação (\%) e IVG, se ajustaram ao modelo quadrático com maiores resultados nas capacidades de retenção de 40 e $50 \%$. O comprimento da parte aérea e o comprimento da raiz também sofreram influência das diferentes capacidades de retenção, onde os maiores resultados foram obtidos aos 40 e $20 \%$ de capacidade de retenção, respectivamente. Já com relação à massa seca e verde, o valor máximo foi observado na capacidade de retenção de $40 \%$. As sementes de Mimosa caesalpiniifolia Benth apresenta alta qualidade fisiológica quando submetidas a capacidades de retenção de 40 e 50\%, contudo são capazes de se adaptar ao estresse hídrico severo, apresentando resultados satisfatórios também aos $20 \%$, podendo assim ser utilizada com sucesso em algumas fases da recuperação de áreas degradadas.

Palavras chave: adaptação, sabiá, semiárido.
\end{abstract}

\section{PHYSIOLOGY AND VIGOR OF SEEDS OF Mimosa caesalpiniifolia BENTH UNDER CONDITIONS OF WATER STRESS}

\begin{abstract}
Mimosa caesalpiniifolia Benth. is a promising species for the implantation of forests in Northeast Brazil. However, water is the factor that influences the germinative process, affecting several physiological processes, such as photosynthesis and respiration, as well as its production. In view of the above, the objective was to evaluate the germination and vigor of seeds of Mimosa caesalpiniifolia Benth. submitted to different water stress conditions. The seeds of M. caesalpiniifolia were collected in the city of. Where they were beneficiated and homogenized for the removal of a representative sample and afterwards they were submitted to the overcoming of dormancy by means of peeling in the region opposite to the micropyle. Soon after, they were disinfested with $0.6 \%$ sodium hypochlorite for 5 minutes and washed in running water for 10 minutes. For the evaluation of the different water stress conditions, the seeds were seeded in individual plastic trays for each replicate, containing sand moistened with five different levels of retention capacity (10, 20, 30, 40, 50 and 60). The variables, germination (\%) and IVG were adjusted to the quadratic model with higher results in retention capacities of 40 and $50 \%$. The shoot length and root length were also influenced by the different retention
\end{abstract}

\footnotetext{
${ }^{1}$ Pós-graduação em Agronomia, Universidade Federal da Paraíba, UFPB, Campus II, Areia - PB. E-mail: acbezerra78@gmail.com

2 Pós-graduação em Horticultura Tropical, Universidade Federal de Campina Grande, UFCG, Campus Pombal, Pombal - PB. E-mail: luanabarbosassb@gmail.com

${ }^{3}$ Pós-graduação em Ciência do solo, Universidade Federal da Paraíba, UFPB, Campus II, Areia-PB. E-mail: jose_flaviocardoso@hotmail.com 4 Docente da Universidade Estadual da Paraíba, UEPB, Campus II, Lagoa Seca - PB. E-mail: camfiraze@bol.com.br
} 
capacities, where the highest results were obtained at 40 and $20 \%$ retention capacity, respectively. Regarding the dry and green mass, the maximum value was observed in the retention capacity of $40 \%$. The seeds of Mimosa caesalpiniifolia Benth present high physiological quality when submitted to retention capacities of 40 and 50\%, however, they are able to adapt to severe water stress, presenting satisfactory results also at $20 \%$, and can therefore be successfully used in recovery phases of degraded areas.

Keywords: adaptation, sabiá, semi-arid.

\section{INTRODUÇÃO}

Mimosa caesalpiniifolia Benth. é uma espécie nativa do Nordeste brasileiro, pertencente à família Fabaceae, conhecida popularmente como sabiá. É uma arbórea pioneira, decídua, heliófila, xerófila, ocorre preferencialmente em solos profundos, tanto em formações primárias como secundárias (Lorenzi, 2008).

Por ser uma espécie pioneira o sabiá é promissor para implantação de florestas no Nordeste brasileiro, pois suas folhas apresentam valor proteico, podendo ser utilizada como fonte de alimento para o gado (Ferreira et al., 2007; Lorenzi, 2008). Além disso, devido ao seu rápido crescimento, possui potencial para recuperação de áreas degradadas, potencial apícola, controle de plantas daninhas e é bastante utilizada como cerca viva em diversas regiões do país (Souza, 2017).

Sementes de espécies que se desenvolvem em solos de regiões áridas e semiáridas, a exemplo de $M$. caesalpiniifolia, comumente encontram condições inadequadas para a germinação, como é o caso dos solos salinos, sódicos ou com déficit hídrico, abundantes nessas regiões (Guedes et al., 2013).

O bioma Caatinga apresenta uma rica biodiversidade com espécies florestais, madeireiras e medicinais adaptadas às condições ambientais desfavoráveis (Gariglio et al., 2010; Siqueira Filho et al., 2012). Contudo, o estudo da influência dessas condições ambientais sobre a fisiologia e a germinação de sementes é bastante importante para preservação, identificação e disseminação das espécies vegetais nativas do Nordeste (Moura et al., 2011).

Fatores como temperatura, disponibilidade de água e luminosidade influenciam no processo germinativo das sementes, entretanto a água é o fator que exerce a mais determinante influência sobre o processo germinativo (Carvalho \& Nakagawa, 2012), pois a reidratação dos tecidos acelera as atividades metabólicas que resultam no fornecimento de energia e nutrientes necessários para a retomada de crescimento do eixo embrionário, além de estar envolvida em todas as outras fases subsequentes do metabolismo da planta (Rego et al., 2011; Ataíde et al., 2014).

Porém, de acordo com Jaleel et al. (2009), o estresse hídrico inibe o crescimento celular reduzindo o desenvolvimento das plantas e afetando diversos processos fisiológicos, como fotossíntese e respiração, bem como a sua produção. Neste sentido, a ação do estresse hídrico sobre o processo germinativo é importante para o entendimento da ecofisiologia da espécie e para avaliar os limites de tolerância e adaptação destas aos fatores ambientais extremos como seca, calor e solos afetados por sais, a semelhança da caatinga e do semiárido nordestino (Guedes et al., 2013).

Diante do exposto, objetivou-se avaliar a germinação e o vigor de sementes de Mimosa caesalpiniifolia Benth. submetidas a diferentes condições de estresse hídrico.

\section{MATERIALE MÉTODOS}

O experimento foi conduzido em ambiente protegido no Centro de Ciências Agrárias e Ambientais da Universidade Estadual da Paraíba, em Lagoa Seca/ $\mathrm{PB}$, situada geograficamente nas coordenadas $\mathrm{S} 7^{\circ}$ 10' 8'’ e W 35 51' 20', Seu clima é o tropical úmido com estação seca de inverno (Aw) de acordo com Köppen e Geiger, com temperatura média anual em torno de $22{ }^{\circ} \mathrm{C}$, sendo a mínima de $14^{\circ} \mathrm{C}$ e a máxima de $33^{\circ} \mathrm{C}$. Localizada no Planalto da Borborema, possui altitude média de $640 \mathrm{~m}$.

As sementes de $M$. caesalpiniifolia foram coletadas na cidade de São Sebastião de Lagoa de Roça/PB, localizada em área de Caatinga paraibana. As sementes foram beneficiadas e homogeneizadas para a retirada de uma amostragem representativa das que seriam utilizadas nos experimentos. Posteriormente as mesmas foram submetidas à superação de dormência por meio de desponte na região oposta à micrópila. Em seguida foram desinfestadas com hipoclorito de sódio a $0,6 \%$, durante 5 minutos e lavadas em água corrente por 10 minutos. 
Para a avaliação das diferentes condições de estresse hídrico, as sementes foram semeadas em bandejas plásticas individuais para cada repetição, contendo areia umedecida com cinco diferentes níveis de capacidade de retenção (10, 20, 30, 40, 50 e 60\%). O delineamento utilizado foi o inteiramente casualizado com quatro repetições de 25 sementes para cada tratamento.

As plântulas de $M$. caesalpiniifolia foram avaliadas quanto as seguintes variáveis: porcentagem de germinação, índice de velocidade de germinação, comprimento da parte aérea e da raiz e massa fresca e seca.

Para a avaliação da porcentagem de germinação, foram realizadas contagens diárias até a estabilização da germinação, seguindo metodologia de Brasil (2009). Em relação ao Índice de velocidade de germinação (IVG) foram feitas contagens diárias do número de plântulas emersas até o final do teste, seguindo a metodologia da equação descrita por Maguire (1962).

$$
\mathrm{IVG}=\frac{G_{1}}{N_{1}}+\frac{G_{2}}{N_{2}}+\cdots+\frac{G_{n}}{N_{n}}
$$

Onde:

G1, G2, Gn = número de plântulas na primeira, na segunda e na última contagem. N1, N2, Nn = número de dias de semeadura à primeira, segunda e última contagem.

O comprimento da parte aérea e da raiz de plântulas foram avaliadas no final do teste de germinação, com plântulas normais de cada repetição, sendo medidas com o auxílio de uma régua graduada em centímetros. A massa verde e seca de plântulas normais foram pesadas em balança analítica com precisão de 0,001g para determinação da massa verde. Estas foram levadas à estufa a $65^{\circ} \mathrm{C}$ até peso constante e posteriormente foram pesadas nas mesmas condições para a determinação da massa seca.

Os dados obtidos foram submetidos à análise de variância, pelo teste F e análise de regressão polinomial, em função das capacidades de retenção, testando o modelo linear e quadrático com auxilio de software estatístico SISVAR (Ferreira, 2000).

\section{RESULTADOS E DISCUSSÃO}

A germinação e o vigor de sementes de $M$. caesalpiniifolia foram influenciadas pelas diferentes capacidades de retenção testada. As variáveis, porcentagem de germinação e IVG, se ajustaram ao modelo quadrático (Figura 1).

A maior percentagem de germinação (88\%) foi observada quando as sementes foram submetidas à capacidade de retenção de $40 \%$ e 50\%. Já com relação ao índice de velocidade de germinação (IVG) o valor máximo $(7,94)$ foi observado na capacidade de retenção de 50\%. Observou-se também que na capacidade de retenção de $60 \%$ houve uma redução acentuada dos resultados nas duas variáveis. Acredita-se que a adaptação da $M$. caesalpiniifolia ao ambiente seco é tanta, que sua exposição ao ambiente com maior disponibilidade hídrica pode reduzir sua germinação e sua velocidade de germinação. De acordo como Marcos Filho (2015), isso ocorre, pois, existe uma grande variação de respostas ao estresse hídrico entre as espécies, desde aquelas muito sensíveis até as mais resistentes, ou seja, a intensidade da resposta germinativa ao estresse hídrico é variável entre sementes de diferentes espécies.

As sementes de $M$. caesalpiniifolia são altamente resistentes ao estresse hídrico severo, pois apresentam altas taxas de germinação nas capacidades de retenção de 10, 20, 30\%. Quando este tipo de estresse é severo, esta situação pode levar à diminuição das defesas da planta, reduzindo o crescimento e a fotossíntese. A adaptabilidade e tolerância ao estresse hídrico são estratégias intrínsecas diferentes nas espécies vegetais, para cada espécie existe um valor de potencial hídrico crítico, abaixo do qual a germinação não ocorre (Pereira et al., 2014; Azerêdo et al., 2016).

Com relação à tolerância de outras sementes da Caatinga ao estresse hídrico, verifica-se que sementes de Myracrodruon urundeuva Fr. All. (Virgens et al., 2012) e Caesalpinia pyramidalis Tul. (Antunes et al., 2011) também são tolerantes ao estresse hídrico, apresentando altas taxas de germinação.

O comprimento da parte aérea e o comprimento da raiz sofreram influência das diferentes capacidades de retenção testadas, onde os dados ajustaram-se ao modelo quadrático (Figura 2). No comprimento da parte aérea as sementes submetidas a $40 \%$ de capacidade de retenção apresentaram maior crescimento $(6,11 \mathrm{~cm})$. E para o comprimento da raiz a capacidade de $20 \%$ foi que apresentou o maior crescimento $(8,09 \mathrm{~cm})$, observando-se grande redução dos resultados no tratamento de $60 \%$ de capacidade de retenção. 
Plantas que crescem sob condições de estresse hídrico tem a habilidade de desenvolver um sistema radicular eficiente assim que a germinação ocorra, visando assegurar um fornecimento de água contínuo para atender à transpiração e ao seu crescimento (Al-Karaki et al., 2007), fato comprovado nessa pesquisa, já que as raízes apresentaram maior crescimento na capacidade de retenção de $20 \%$.

Para as variáveis massa verde e seca das plântulas de $M$. caesalpiniifoli ajustaram-se ao modelo quadrático, onde a massa verde e seca apresentou valor máximo $(0,085 \mathrm{~g}$ e $0,009 \mathrm{~g}$, respectivamente) na capacidade de retenção de $40 \%$ (Figura 3). Assim como os resultados
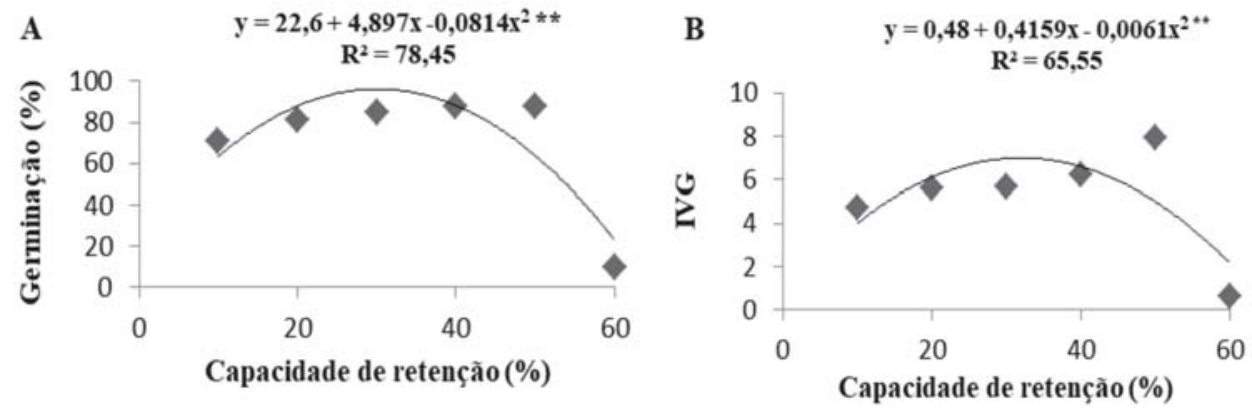

Figura 1 - Porcentagem de germinação (A) e índice de velocidade de germinação (B), de sementes de Mimosa caesalpiniifolia Benth. submetidas a diferentes níveis de capacidade de retenção do solo.
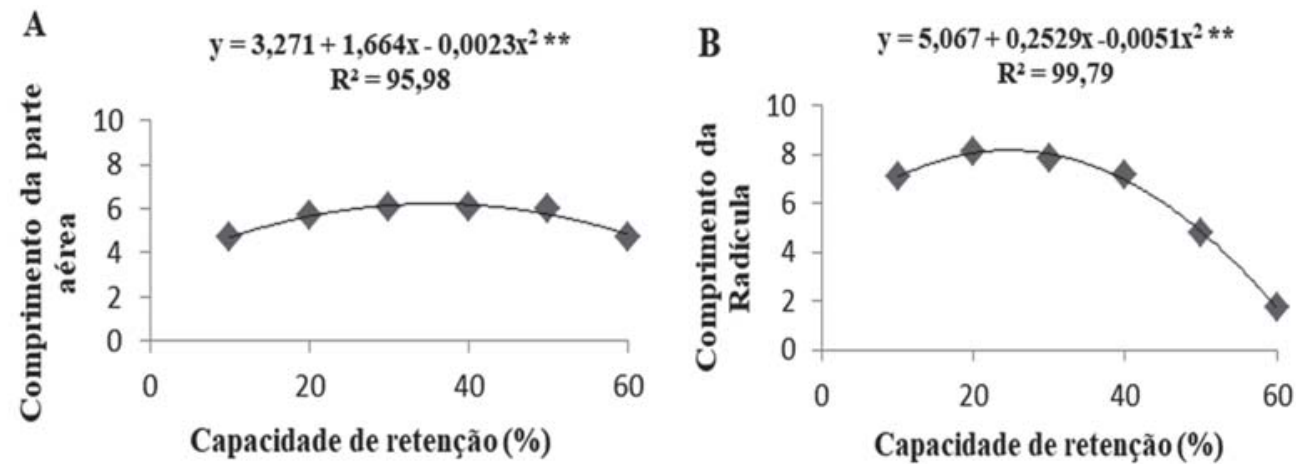

Figura 2 - Comprimento da parte aérea (A) e comprimento da radícula (B), de plântulas de Mimosa caesalpiniifolia Benth. submetidas a diferentes níveis de capacidade de retenção do solo.
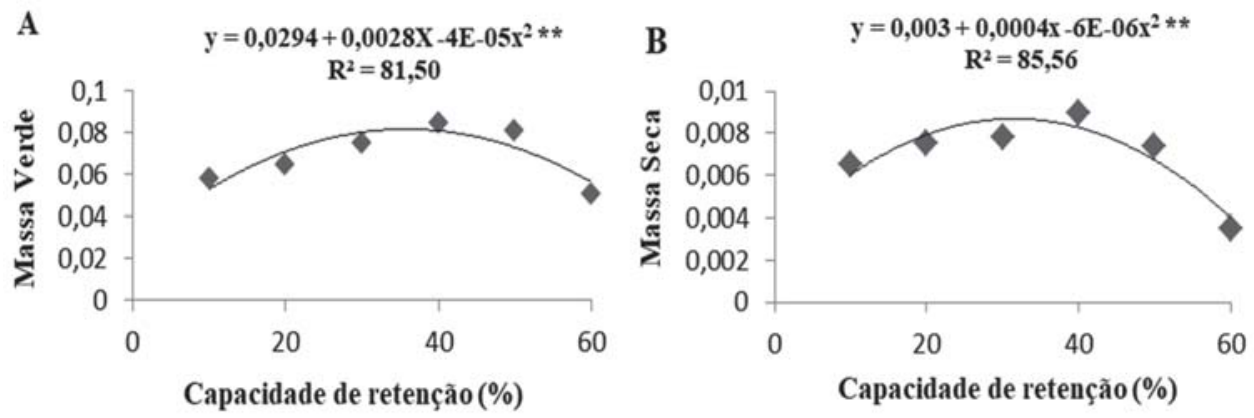

Figura 3 - Massa verde (A) e massa seca (B), de plântulas de Mimosa caesalpiniifolia Benth. submetidas a diferentes níveis de capacidade de retenção do solo. 
anteriores, aos $60 \%$ de capacidade de retenção, houve redução das variáveis analisadas.

O baixo acúmulo de matéria verde e seca nas capacidades de retenção de 10, 20 e $30 \%$ nas sementes de $M$. caesalpiniifolia, pode ser explicado pela redução na expansão celular causada pelo estresse hídrico (Taiz et al., 2017). Além disso, essa redução na biomassa seca, assim como no crescimento das plântulas, pode ser explicada pela diminuição no metabolismo das sementes, em função da menor disponibilidade de água para digestão das reservas e translocação de produtos metabolizados (Gordin et al., 2014; Gordin et al., 2015).

\section{CONCLUSÃO}

As sementes de Mimosa caesalpiniifolia Benth apresentam alta qualidade fisiológica quando submetidas a capacidades de retenção de 40 e 50\%, contudo, são capazes de se adaptar ao estresse hídrico severo, apresentando resultados satisfatórios também aos 20\%, podendo assim, ser utilizada com sucesso em algumas fases da recuperação de áreas degradadas.

\section{REFERÊNCIAS}

AL-KARAKI, G.N.; AL-AJMI, A.; OTHMAN, Y. Seed germination and early root growth barley cultivars as affected by temperature and water stress. American-Eurasian Journal Agricultural and Environmental Science, Dubai, v.2, n.2, p.112-117, 2007.

ANTUNES, C.G.C.; PELACANI, C.R.; RIBEIRO, R.C. et al. Germinação de sementes de Caesalpinia pyramidalis Tul. (catingueira) submetidas à deficiência hídrica. Revista Árvore, Viçosa, v.35, n.5, p.1007-1015, 2011.

ATAÍDE, G.M.; BORGES, E.E.L.; FLORES, A.V. et al. Avaliação preliminar da embebição de sementes de jacarandá-da-bahia. Pesquisa Florestal Brasileira, v.34, n.78, p.133-139, 2014.

AZERÊDO, G.A.; PAULA, R.C.; VALERI, S.V. Germination of Piptadenia moniliformis Benth seeds under water stress. Ciência Florestal, v.26, n.1, p.193-202, 2016.

BRASIL, Ministério da Agricultura e Reforma Agrária. Regras para análise de sementes. Brasília, 2009. 399p.
CARVALHO, M.N.; NAKAGAWA, J. Sementes: ciência, tecnologia e produção. 5.ed. Jaboticabal: FUNEP, 2012. 590p.

CARVALHO, C.J.R. Respostas de plantas de Schizolobium amazonicum (S. parahyba var. amazonicum) e Schizolobium parahyba (Schizolobium parahybum) à deficiência hídrica. Revista Árvore, Viçosa, v.29, n.6, p.907-914, 2005.

FERREIRA, D.F. Análise estatística por meio do SISVAR (Sistema para Análise de Variância) para Windows versão 4.0. In: REUNIÃO ANUAL DA REGIÃO BRASILEIRA DA SOCIEDADE INTERNACIONAL DE BIOMETRIA, 45., 2000, São Carlos. Anais... São Carlos: UFSCar, p.255-258, 2000.

FERREIRA, R.L.C.; LIRA JÚNIOR, M.A.; ROCHA, M.S. et al. Deposição e acúmulo de matéria seca e nutrientes em serapilheira em bosque de Sabiá (Mimosa caesalpiniaefolia Bent.). Revista Árvore, Viçosa, MG, v.31, n.1, p.7-12, 2007.

GARIGLIO, M.A.; SAMPAIO, E.V.S.B.; CESTARO, L.A. et al. Uso sustentável e conservação dos recursos florestais da Caatinga. Brasília: Serviço Florestal Brasileiro, 2010. 368 p.

GORDIN, C.R.B.; MARQUES, R.F.; MASETTO, T.E. et al. Temperaturas e disponibilidades hídricas do substrato na germinação de sementes de niger. Bioscience Journal, v.30, n.3, p.112118, 2014.

GORDIN, C.R.B.; SCALON, S.P.Q.; MASETTO, T.E. Disponibilidade hídrica do substrato e teor de água da semente na germinação de niger.

Pesquisa Agropecuária Tropical, v.45, n.3, p.312-318, 2015.

GUEDES, R.S.; ALVES, E.U.; VIANA, J.S. et al. Germinação e vigor de sementes de Apeiba tibourbou submetidas ao estresse hídrico e diferentes temperaturas. Ciência Florestal, v.23, n.1, p.45-53, 2013.

JALEEL, C.A.; MANIVANNAN, P.; WAHID, A. et al. Drought Stress in Plants: A Review on morphological chacacteristics and pigments composition. International Journal Agricultural Biology, Islamabad, v.11, p.100105, 2009. 
LORENZI, H. Árvores brasileiras: manual de identificação e cultivo de plantas arbóreas nativas do Brasil. 5.ed. Nova Odessa: Instituto Plantarum, 2008. v.1. 368p.

MAGUIRE, J.D. Speed of germination-aid in selection and evaluation for seeding emergence and vigor. Crop Science, v.2. p.176-177, 1962.

MARCOS FILHO, J. Fisiologia de sementes de plantas cultivadas. 2.ed. Londrina: Abrates, 2015. 660p.

MOURA, M.R.; LIMA, R.P.; FARIAS, S.G.G. et al. Efeito do estresse hídrico e do cloreto de sódio na germinação de Mimosa caesalpiniifolia Benth.

Revista Verde de Agroecologia e Desenvolvimento Sustentável, v.6, n.2, p.230-235, 2011.

PEREIRA, M.R.R.; MARTINS, C.C.; MARTINS, D. et al. Estresse hídrico induzido por soluções de PEG e de $\mathrm{NaCl}$ na germinação de sementes de nabiça e fedegoso. Bioscience Journal, v.30, n.3, p.687-696, 2014.
REGO, S.S.; FERREIRA, M.M.; NOGUEIRA, A.C. et al. Estresse hídrico e salino na germinação de sementes de Anadenanthera colubrina (Veloso) Brenan. Journal of Biotechnology and Biodiversity, Gurupi, v.2, n.4, p.37-42, 2011.

SIQUEIRA FILHO, J.A. Flora das caatingas do rio São Francisco. História Natural e Conservação. Rio de Janeiro; Andrea Jacbsson, 2012.552p.

SOUZA, E.C. Germinação e vigor de sementes de Mimosa caesalpiniifolia Benth. sob estresse hídrico e salino. 2017. 63p. Dissertação (Mestrado) - Programa de Pósgraduação em Ambiente, Tecnologia e Sociedade, Universidade Federal Rural do Semiárido, 2017.

TAIZ, L.; ZEIGER, E.; MOLLER, I.M. et al. Fisiologia e desenvolvimento vegetal. 6.ed. Porto Alegre: ARTMED, 2017. 888p.

VIRGENS, I.O.; CASTRO, R.D.; FERNANDEZ, L.G. et al. Comportamento fisiológico de sementes de Myracrodruon urundeuva Fr. All. (Anacardiaceae) submetidas a fatores abióticos. Ciência

Florestal, Santa Maria, v.22, n.4, p.681-692, 2012.

Recebido para publicação em 1/1/2019 e aprovado em 25/3/2019. 